\title{
Parameters predicting postoperative unilateral disease in patients with unilateral prostate cancer in diagnostic biopsy: a rationale for selecting hemiablative focal therapy candidates
}

\author{
Stavros Sfoungaristos, MD; Petros Perimenis, MD
}

Patras University Hospital, Urology Department, Patras, Greece

Cite as: Can Urol Assoc J 2013;7:E82-E87. http://dx.doi.org/10.5489/cuaj.268. Epub 2011 November 2.

\section{Abstract}

Background: Focal hemiablative therapy for prostate cancer is a new treatment alternative. Unilateral and unifocal disease are its main limitations. The aim of this study was to identify the epidemiological, clinical and pathological parameters that may predict unilateral disease in patients diagnosed with prostate cancer.

Methods: We performed a retrospective analysis of patients at our institution between January 2005 and January 2011. Only patients with unilateral disease in prostate biopsy were part of the study. The analysis included age, preoperative prostate-specific antigen (PSA) and its density, prostate volume, biopsy first and second Gleason pattern and Gleason summary, number of biopsy cores, percentage of cancer in biopsy material and the presence of highgrade prostatic intraepithelial neoplasia. Their role as potential predictors was evaluated by univariate and multivariate analysis. Results: A total of 161 patients had unilateral disease after prostate biopsy. A significant correlation was found between prostate volume, PSA density and percentage of cancer in biopsy material and the presence of unilateral disease in the surgical specimen. These are the same factors significant in the univariate analysis. The results of the multivariate analysis demonstrated that PSA density $(p=0.015)$ and percentage of cancer in biopsy material $(p=0.028)$ are the most significant predictors.

Interpretation: Our results demonstrate that PSA density and the percentage of cancer in biopsy cores are significant predictors for prostate cancer unilaterality and should be considered for the selection of hemiablative focal therapy candidates.

$\mathrm{T}$ he therapeutic concept of focal therapy (FT) consists in targeting and destroying the malignant part of the suffered organ, while preserving the normal tissue. Focal therapy, which aims to preserve healthy, non-malignant prostatic areas, is considered an alternative treatment for prostate cancer. Organ-sparing treatment may benefit functional parameters mostly affected by radical treatments, like continence and potency. ${ }^{1,2}$
Candidates for hemiablative therapy are those patients with unifocal lesions and unilateral disease or patients with bilateral cancer and insignificant disease contralaterally. Even though prostate cancer is known as a multifocal disease, contemporary studies report an increase in frequency of cancer unifocality from $13 \%$ to $38 \%$ and unilaterality from $19 \%$ to $63 \% .^{3-5}$ Focal therapy has gained a place in the group of potential prostate cancer treatment modalities and several studies are working on the efficacy and reliability of this therapeutic option. Focal therapy may provide sufficient oncological results and minimize postoperative morbidity, although long-term cancer specific survival data are missing.

Appropriate patient selection is the cornerstone for FT success. An inadequate estimation of disease may lead to incomplete and limited cancer control. In the absence of well-standardized epidemiological, clinical, pathological and imaging parameters that can predict tumour status, we relied on prostate biopsy findings to estimate disease extension and aggressiveness. ${ }^{6}$ However, conventional biopsy schemes have been shown to be unreliable in identifying appropriate patients for FT. ${ }^{7,8}$ Several novel imaging techniques, including contrast enhanced ultrasound and magnetic resonance biopsy, have attempted to better define and visualize prostate cancer within the gland. ${ }^{9,10}$ Even though the results are promising, they need validation before being introduced to the clinical setting. Three-dimensional transperineal mapping biopsies are promising, since they can be used either as a selection tool for FT candidates by identifying cancer location and extension within the prostate, or as a guide for the FT procedure. ${ }^{11}$ However, hospitalization and general anesthesia are needed and, therefore, its use is limited.

Considering the limitations of currently used imaging techniques to identify appropriate FT patients, we sought to analyze and identify the epidemiological, clinical and pathological parameters that may predict prostate cancer unilaterality and, consequently, help us to select candidates for FT. 


\section{Patients and methods}

After we obtained approval by the Ethics committee of our institution, we performed a retrospective analysis of patients who underwent an open or laparoscopic radical prostatectomy for clinically localized prostate cancer from January 2005 until January 2011. Their records, regarding transrectal ultrasound-guided prostate biopsy, were evaluated. Patients who had been diagnosed with unilateral prostate cancer were included in the study. Unilateral disease was defined as malignancy found only in cores of one of the prostate lobes. Patients found to have cancer material in cores from both lobes (bilateral disease) were excluded. We excluded patients with any preoperative therapy for prostate cancer (active surveillance, hormone therapy, radiation therapy), patients found to have not localized disease preoperatively, in terms of extracapsular extension, seminal vesicle invasion and lymph nodes metastases, and patients in whom the diagnosis was established after transurethral resection of the prostate. Patients with incomplete medical records were also excluded.

The analysis comprised patients' age, preoperative prostate-specific antigen (PSA), prostate volume, PSA density, biopsy first and second Gleason pattern and Gleason summary, the number of the biopsy cores, the percentage of cancer in biopsy material (PCBM), by means of proportion of cancer out of all cores examined, and the presence of high-grade prostatic intraepithelial neoplasia (HGPIN). The PSA was measured before any prostate manipulation, such as digital rectal examination, transrectal ultrasound or biopsy. Transrectal ultrasound biopsy was performed after local anesthesia and at least 6 cores from both lobes were obtained. To preserve the quality of prostate biopsy results, we applied the Vienna nomogram for patients with PSA between 2 to $10 \mathrm{ng} / \mathrm{mL}$, while the standard 12 or more cores biopsy was applied in patients with preoperative PSA $>10 \mathrm{ng} / \mathrm{mL}$. All cores were examined by expert pathologists from our institution and the results of the cancer grade and the proportion of cancer from all cores (PCBM) were reported.

An open retropubic or laparoscopic extraperitoneal radical prostatectomy was performed in all patients by 4 experienced surgeons. The surgical specimen was sent for pathological examination and evaluation was done by slicing the specimen in serial sections at regular intervals of $3 \mathrm{~mm}$. Finally, a histological report on the prostate dimensions and pathological stage was obtained. The 2009 TNM (Tumour Node Metastasis) classification for prostate cancer was used to classify the pathological stage. Pathological unilateral disease was defined as localized disease in one prostatic lobe with no extracapsular extension and no seminal vesicle or lymph node involvement. According to the information of the maximum transverse diameter (D1), the maximum anteroposterior diameter (D2) and the maximum longitudinal diameter (D3) obtained by the pathologoanatomic report, the pathological prostate volume was calculated by using the prostate ellipse dimension theory formula $(\mathrm{D} 1 \times \mathrm{D} 2 \times \mathrm{D} 3 \times \pi / 6)$. The PSA density was calculated by dividing the preoperative PSA value and prostate volume. Even though prostate volume was calculated postoperatively according to the pathological prostate dimensions, there is a high positive correlation between preoperative (during transrectal ultrasound) and postoperative calculation of prostate volume. $^{12}$

Statistical analysis was performed by using SPSS version 17 (SPSS Inc, Chicago, IL). The descriptive statistics are presented as the mean \pm standard deviation (SD) and interquartile range (IQR) for continuous variables and as the absolute and percent frequency for categorical variables. The normality condition of the numerical variables was analyzed using the Kolmogorov-Smirnov test. None of them had normal distribution. For this reason, the MannWhitney test was used to compare means between numerical groups. The Chi-square $\chi 2$ test was used for categorical variables. A univariate analysis was performed to identify the predictive significance of age, preoperative PSA, prostate volume, PSA density, preoperative first and second Gleason pattern, Gleason summary, number of biopsy cores, PCBM and the presence of HGPIN in biopsy cores in prediction of prostate cancer unilaterality. A multivariate analysis was also performed for the variables identified as statistically important in univariate analysis, using logistic regression. Prostate volume, PCBM and patient' age were evaluated as continuous variables. The other parameters entered the univariate and multivariate analyses as binary variables. The optimal cutoff values, sensitivity and specificity for quantitative variables (which were significant predictors for pathological unilaterality in multivariate analysis) were estimated by using receiver operating curve (ROC) analysis. Positive [true positive/(true positive+false positive)] and negative predictive value [true negative/(true negative+false negative)] were also estimated. All tests were 2 -tailed with $p<0.05$ considered statistically significant.

\section{Results}

Within the study period, 409 patients underwent a radical prostatectomy. There were insufficient data, regarding biopsy results, in 112 patients; consequently, the records of 297 were evaluated. From this cohort, 161 patients (54.2\%) were found to have unilateral prostate cancer in biopsy results and were entered in our analysis. The median age of patients was 67 years. The median preoperative PSA value was $8.2 \mathrm{ng} / \mathrm{mL}$ and the median prostate volume was $40 \mathrm{~mL}$. The PSA density ranged from 0.06 to $1.85 \mathrm{ng} / \mathrm{mL}^{2}$ (Table 1 ). The Median PCBM was $18.5 \%$. The pathological stage was also tallied (Table 1). Lymph node dissection was performed 


\begin{tabular}{|c|c|}
\hline \multicolumn{2}{|l|}{ Characteristics } \\
\hline No. patients & 161 \\
\hline \multicolumn{2}{|l|}{ Age (years) } \\
\hline Mean \pm SD, IQR & $66.5 \pm 6.6,10$ \\
\hline \multicolumn{2}{|c|}{ Prostate volume $(\mathrm{mL})$} \\
\hline Mean \pm std, IQR & $44.0 \pm 22.1,27$ \\
\hline \multicolumn{2}{|l|}{ PSA (ng/mL), n (\%) } \\
\hline$<10$ & $106(65.8)$ \\
\hline$\geq 10$ & $55(34.2)$ \\
\hline \multicolumn{2}{|c|}{ PSAD (ng/mL $\left.{ }^{2}\right), n(\%)$} \\
\hline$\leq 0.2$ & $80(49.7)$ \\
\hline$>0.2$ & $81(50.3)$ \\
\hline \multicolumn{2}{|c|}{ No. biopsy cores, n (\%) } \\
\hline$\leq 12$ & $78(48.4)$ \\
\hline$>12$ & $83(51.6)$ \\
\hline \multicolumn{2}{|l|}{ РCBM (\%) } \\
\hline Mean $\pm S D$, IQR & $25.9 \pm 23.8,30$ \\
\hline \multicolumn{2}{|l|}{ Biopsy GS, n (\%) } \\
\hline$\leq 6$ & $80(49.7)$ \\
\hline$\geq 7$ & $81(50.3)$ \\
\hline \multicolumn{2}{|l|}{$1^{\text {st }}$ pattern, n (\%) } \\
\hline$\leq 3$ & $114(70.8)$ \\
\hline$\geq 4$ & $47(29.2)$ \\
\hline \multicolumn{2}{|l|}{$2^{\text {nd }}$ pattern, n (\%) } \\
\hline$\leq 3$ & $111(68.9)$ \\
\hline$\geq 4$ & $50(31.1)$ \\
\hline \multicolumn{2}{|l|}{ HGPIN, n (\%) } \\
\hline No & $75(46.6)$ \\
\hline Yes & $86(53.4)$ \\
\hline \multicolumn{2}{|c|}{ Pathological stage, n (\%) } \\
\hline $\mathrm{T} 2 \mathrm{a}$ & $29(18.0)$ \\
\hline $\mathrm{T} 2 \mathrm{~b}$ & $23(14.3)$ \\
\hline $\mathrm{T} 2 \mathrm{c}$ & $59(36.6)$ \\
\hline Т3a & $28(17.4)$ \\
\hline T3b & $20(12.4)$ \\
\hline $\mathrm{T} 4$ & $2(1.2)$ \\
\hline $\mathrm{N}+$ & $19(11.8)$ \\
\hline$\leq \mathrm{T} 2 \mathrm{~b}$ N1 & 7 \\
\hline$\geq \mathrm{T} 3 \mathrm{a} \mathrm{N} 1$ & 12 \\
\hline
\end{tabular}

SD: standard deviation; IQR: interquartile range; GS: Gleason score; PSA: prostate-specific antigen; PSAD: PSA density; PCBM: percentage of cancer in biopsy material;

HGPIN: high-grade prostatic intraepithelial neoplasia, $\mathrm{N}+$ : lymph node invasion.

in 133 patients and invasion was found in 19 patients (7 of these 19 patients had malignancy in one prostate lobe).

Finally, pathological unilateral prostate cancer was confirmed in $45(28.0 \%)$ patients after their specimen analysis from their radical prostatectomy. In fact, $29(18.0 \%)$ and $23(14.3 \%)$ patients had pathological T2a and T2b disease, respectively. However, 7 of them had lymph node invasion after the pathological evaluation of the surgical specimen and were not considered unilateral since they did not have localized prostate cancer. The clinical and pathological characteristics of these patients were tallied (Table 2).

There was a significant correlation between unilateral disease and PSA density $(p<0.001)$ and PCBM $(p=0.005)$. The same variables found to be significant predictors for unilateral prostate cancer in univariate analysis (Table 3). In multivariate analysis, PSA density $\leq 0.2 \mathrm{ng} / \mathrm{mL} 2$ and PCBM were the most significant predictors with $p$ values of 0.004 and 0.027 , respectively (Table 3 ). The estimated optimal cutoff value of PCBM was $\leq 35 \%$, obtained by ROC analysis (area under the curve $=0.643$ ). We also noted the predictive parameters of PSA density and PCBM (Table 4).

\section{Discussion}

The introduction of PSA and transrectal ultrasound in prostate cancer screening has led to an increase in the detection of indolent, non-significant cancer..$^{13,14}$ This shift in prostate cancer epidemiology and diagnosis has forced the re-examination of the standard radical therapies, such as radiation therapy and prostatectomy. Given that many patients are now diagnosed early and most of them suffer from low-malignant potential cancer and will not die from the disease, conservative treatment strategies, such as active surveillance and watchful waiting, have been introduced. The rationale for deferred treatment modalities is the preservation of potency and continence while offering, by a close follow-up, an appropriate treatment if necessary. However, patients' anxiety is often a clinical obstacle for such treatments. ${ }^{15}$

Focal therapy has been introduced as an alternative bridging radical and deferred therapeutical options. Partial organ ablation can be offered for patients with insignificant cancer to preserve the non-malignant part of the prostate gland and minimize any functional side effects. Tumour cryoablation and high intensity focused ultrasound are well-known ablative techniques of FT and have been studied in the clinical setting. ${ }^{16,17}$ Laser photothermal therapy and irreversible electroporation have been recently proposed as reliable alternatives, however their use is still under investigation. ${ }^{18,19}$ The efficacy of FT has not been well-documented because of immature collective experience and relatively small patient cohorts. Short-term control rates of $55 \%$ to $84 \%$ have been reported in high intensity focused ultrasound case series, ${ }^{20}$ while success rates of $88 \%$ to $94 \%$ have been noticed in cryoablation studies, based on postoperative PSA stability after about 2 years of follow-up.,21

The ultimate success and applicability of FT relies mainly on preoperative patient selection and optimal tumour characterization to maximize cancer control and minimize morbidity. Since unifocal and unilateral cancer is the cornerstone for hemiablative FT implementation, several studies have evaluated the clinical and pathological parameters that can identify appropriate candidates. 
Table 2. Correlation of clinical and pathological variables with unilaterality after pathological evaluation of the prostate specimen

\begin{tabular}{|c|c|c|c|}
\hline Characteristics & Unilateral PC & Bilateral PC & $p$ \\
\hline No. patients (\%) & $45(28.0)$ & $116(72.0)$ & \\
\hline Age (years) & & & $0.156^{\dagger}$ \\
\hline Mean \pm SD, IQR & $65.5 \pm 6.1,9$ & $66.9 \pm 6.7,9$ & \\
\hline Prostate volume (mL) & & & $0.010^{\dagger}$ \\
\hline Mean $\pm S D, I Q R$ & $51.1 \pm 24.7,34$ & $41.0 \pm 20.4,23$ & \\
\hline PSA (ng/mL), n (\%) & & & $0.105^{\ddagger}$ \\
\hline$<10$ & $34(32.1)$ & $72(67.9)$ & \\
\hline$\geq 10$ & $11(20.0)$ & $44(80.0)$ & \\
\hline PSAD (ng/mL $\left.{ }^{2}\right), n(\%)$ & & & $<0.001^{\ddagger *}$ \\
\hline$\leq 0.2$ & $33(41.3)$ & $47(58.8)$ & \\
\hline$>0.2$ & $12(14.8)$ & $69(85.2)$ & \\
\hline No. of biopsy cores, $\mathrm{n}(\%)$ & & & $0.527^{\ddagger}$ \\
\hline$\leq 12$ & $20(25.6)$ & $58(74.4)$ & \\
\hline$>12$ & $25(30.1)$ & $58(69.9)$ & \\
\hline PCBM (\%) & & & $0.005^{\dagger *}$ \\
\hline Mean $\pm S D, I Q R$ & $16.7 \pm 14.7,19$ & $29.5 \pm 25.7,32$ & \\
\hline Biopsy GS, n (\%) & & & $0.899^{\ddagger}$ \\
\hline$\leq 6$ & $22(27.5)$ & $58(72.5)$ & \\
\hline$\geq 7$ & $23(28.4)$ & $58(71.6)$ & \\
\hline $1^{\text {st }}$ pattern, n (\%) & & & $0.226^{\ddagger}$ \\
\hline$\leq 3$ & $35(30.7)$ & 79 (69.3) & \\
\hline$\geq 4$ & $10(21.3)$ & 37 (78.7) & \\
\hline $2^{\text {nd }}$ pattern, $\mathrm{n}(\%)$ & & & $0.442^{\ddagger}$ \\
\hline$\leq 3$ & $29(26.1)$ & $82(73.9)$ & \\
\hline$\geq 4$ & $16(32.0)$ & $34(68.0)$ & \\
\hline HGPIN, n (\%) & & & $0.285^{\ddagger}$ \\
\hline No & $24(32.0)$ & $51(68.0)$ & \\
\hline Yes & $21(24.4)$ & $65(75.6)$ & \\
\hline
\end{tabular}

Recent studies which evaluated the agreement between tumour laterality in biopsy cores and laterality in pathological analysis of the specimen of radical prostatectomy have reported conflicting results. In a recent analysis of 590 patients with unilateral disease in their preoperative biopsy, $27.3 \%$ had unilateral confirmation after radical prostatectomy. ${ }^{22}$ Similar results have been reported from the SEARCH database. ${ }^{23}$ Out of 261 men with low-risk prostate cancer, defined as clinical stage T1c or T2a, PSA $<10 \mathrm{ng} / \mathrm{mL}$, Gleason score $\leq 6$ and only 1 or 2 ipsilateral positive cores on at least sextant biopsy, only 93 (35.1\%) had unilateral disease following examination of radical prostatectomy specimens. Coming from the opposite perspective, the authors of another analysis of 1184 radical prostatectomy specimens have reported that 1 out of 5 patients had unilateral, small volume prostate cancer that could be treated with $\mathrm{FT} .{ }^{5}$ Our analysis revealed that only 45 (28\%) out of 161 studied patients with preoperative unilateral prostate cancer, based on biopsy findings, had unilateral disease after radical pros- tatectomy specimens evaluation. These results confirm that, by using current biopsy schemes, only a small percentage of patients can be correctly identified with unilateral prostate cancer; other preoperative parameters are mandatory for better patients' selection.

In the present study, an increased number of obtained cores $(>12)$ during transrectal ultrasound biopsy failed to improve the detection rate of pathological unilateral disease and no statistically significant difference was shown between patients who underwent a $\leq 12$-core prostate biopsy or a more extended procedure. Inadequate identification of unilateral cancers was a major clinical problem in the FT implementation and, for this reason, new imaging modalities and biopsy techniques have been evaluated to better map prostate cancer within the gland. ${ }^{9-11}$ Although the results are interesting, the efficacy, safety and superiority compared to conventional imaging and biopsy schemes will need to be determined before these approaches become standard.

In the absence of well-standardized imaging criteria to 


\begin{tabular}{|c|c|c|c|c|}
\hline \multicolumn{5}{|c|}{ Univariate analysis } \\
\hline & \multirow[b]{2}{*}{ Sig. } & \multirow[b]{2}{*}{$\operatorname{Exp}(B)$} & \multicolumn{2}{|c|}{ 95\% Cl for EXP(B) } \\
\hline & & & Lower & Upper \\
\hline Age & 0.237 & 0.969 & 0.920 & 1.021 \\
\hline PSA & 0.108 & 1.899 & 0.869 & 4.106 \\
\hline PSAD & $0.000 *$ & 4.037 & 1.893 & 8.612 \\
\hline Biopsy cores & 0.527 & 0.800 & 0.401 & 1.597 \\
\hline PCBM & $0.003^{*}$ & 0.971 & 0.951 & 0.990 \\
\hline GS & 0.899 & 0.957 & 0.480 & 1.904 \\
\hline $1^{\text {st }}$ pattern & 0.228 & 1.639 & 0.734 & 3.663 \\
\hline $2^{\text {nd }}$ pattern & 0.443 & 0.752 & 0.362 & 1.559 \\
\hline HGPIN & 0.286 & 0.687 & 0.344 & 1.370 \\
\hline \multicolumn{5}{|c|}{ Multivariate analysis } \\
\hline & & & \multicolumn{2}{|c|}{ 95\% Cl for EXP(B) } \\
\hline & Sig. & $\operatorname{Exp}(B)$ & Lower & Upper \\
\hline PSAD & $0.004^{*}$ & 3.209 & 1.466 & 7.022 \\
\hline PCBM & $0.027^{*}$ & 0.977 & 0.958 & 0.997 \\
\hline
\end{tabular}

safely identify appropriate patients for FT, data from imaging techniques should be combined with clinical and pathological criteria before FT is proposed to a patient. In a contemporary clinical series of unilateral cryoablation, selection criteria are mainly preoperative PSA, clinical stage, biopsy Gleason score and biopsy findings, regarding tumour involvement and the number of contiguous cores. ${ }^{21,24}$ In some series, mandatory inclusion criteria are negative neurovascular bundle and seminal vesicle biopsies when extracapsular extension of the disease is suspected. ${ }^{2}$

In a large study of 1114 patients with unilateral disease, based on the biopsy findings, the authors reported that the presence of HGPIN on biopsy significantly predicts contralateral prostate lobe involvement and should be considered an exclusion criterion for $\mathrm{FT}^{25}$ This was not the case in our study; HGPIN was not involved in the prediction of unilaterality after radical prostatectomy.

The PSA density has been reported to correlate with unilateral prostate cancer prediction in some case series. Actually, a recent study of 438 patients reported that, using a cutoff value of $<0.056 \mathrm{ng} / \mathrm{mL}^{2}$, PSA density can be reliably used to select patients for FT. ${ }^{26}$ It is of high interest that, in our cohort, none of the analyzed patients had a PSA density lower than this cutoff point.
We analysed preoperative PSA values and biopsy Gleason score as binary variables, regarding values that have been reported to characterize low-risk cancer (PSA $<10 \mathrm{ng} / \mathrm{mL}$ and Gleason score $\leq 6)$. None of these cut-off values were significant in predicting pathologic unilateral prostate cancer in patients primarily diagnosed with unilateral prostate cancer during biopsy. Our results demonstrated that PSA density, with a cutoff value of $\leq 0.2 \mathrm{ng} / \mathrm{mL}^{2}$, was the strongest predictor for pathological unilateral disease $(p=0.004)$ in multivariate analysis with a sensitivity of $73.3 \%$ and a negative predictive value of $85.2 \%$.

Furthermore, our study revealed the value of PCBM to predict unilateral prostate cancer. A cutoff value of $\leq 35 \%$ was optimal. It is of great interest that all 39 patients who had a PSA density of $\leq 0.2 \mathrm{ng} / \mathrm{mL}^{2}$ and PCBM $\leq 35 \%$ had unilateral prostate cancer confirmed by radical prostatectomy.

Our study has some important limitations. Apart of its retrospective nature, the study included prostate biopsy procedures by different operators. This resulted in missing prebiopsy imaging evidence that could be valuable for patient selection and categorization. Furthermore, we believe that inter-operator variability in obtaining prostate cores may influence the preoperative disease stratification as unilateral or bilateral. The results would be more robust if all biopsy

Table 4. Sensitivity, specificity, positive and negative predictive value of PSA density $\geq 0.2 \mathrm{ng} / \mathrm{ml}^{2}$ and percentage of cancer in biopsy material $\leq \mathbf{3 5} \%$ for pathological unilateral prostate cancer prediction

\begin{tabular}{lllll} 
& Sensitivity & Specificity & PPV & NPV \\
PSAD $\leq 0.2$ & $73.3 \%$ & $59.5 \%$ & $41.3 \%$ & $85.2 \%$ \\
PCBM $\leq 35 \%$ & $88.9 \%$ & $31.9 \%$ & $33.6 \%$ & $88.1 \%$ \\
\hline
\end{tabular}

PSAD: prostate-specific antigen density; PCBM: percentage of cancer in biopsy material; PPV: positive predictive value; NPV: negative predictive value. 
procedures were performed in 1 institution by 1 operator. We also used pathological prostate volume as a surrogate of preoperative prostate volume. Consequently, PSA density was calculated based on the postoperative prostate volume. However, several reports have confirmed that preoperative, based on transrectal ultrasound, and postoperative prostate volume are significantly correlated..$^{12}$ It would be essential to demonstrate our PSA density and PCBM cutoff points in more patients.

\section{Conclusion}

A great proportion of patients initially diagnosed by biopsy with unilateral prostate cancer actually have bilateral disease in prostatectomy specimens. Therefore, it is important to evaluate other preoperative clinical and pathological parameters to correctly identify unilaterality. Our results demonstrate that PSA density and the percentage of cancer in biopsy cores are reliable and significant predictors for prostate cancer unilaterality after radical prostatectomy and should be considered in the selection of appropriate patients for FT.

Competing interests: None declared.

This paper has been peer-reviewed.

\section{References}

1. Ellis DS, Manny TB Jr, Rewcastle JC. Focal cryosurgery followed by penile rehabilitation as primary treatment for localized prostate cancer: initial results. Urology 2007;70:9-15.

2. Onik G, Vaughan D, Lotenfoe R, et al. "Male lumpectomy": focal therapy for prostate cancer using cryoablation. Urology 2007;70:16-21.

3. Cheng L, Jones TD, Pan CX, et al. Anatomic distribution and pathologic characterization of small-volume prostate cancer $(<0.5 \mathrm{ml})$ in whole-mount prostatectomy specimens. Mod Pathol 2005;18:1022-6.

4. Arora R, Koch MO, Eble JN, et al. Heterogeneity of Gleason grade in multifocal adenocarcinoma of the prostate. Cancer 2004; 100:2362-6.

5. Mouraviev V, Mayes JM, Sun L, et al. Prostate cancer laterality as a rationale of focal ablative therapy for the treatment of clinically localized prostate cancer. Cancer 2007;110:906-10.
6. Polascik TJ, Mayes JM, Schroeck FR, et al. Patient selection for hemiablative focal therapy of prostate cancer: variables predictive of tumor unilaterality based upon radical prostatectomy. Cancer 2009;115:2104-10.

7. Mayes JM, Mouraviev V, Sun L, et al. Can the conventional sextant prostate biopsy accurately predict unilateral prostate cancer in low-risk, localized, prostate cancer? Urol Oncol 2011;29:166-70.

8. Tsivian M, Kimura M, Sun L, et al. Predicting unilateral prostate cancer on routine diagnostic biopsy: sextant vs extended. BJU Int 2009 Oct 10. [Epub ahead of print].

9. Seitz M, Gratzke C, Schlenker B, et al. Contrast-enhanced transrectal ultrasound (CE-TRUS) with cadencecontrast pulse sequence (CPS) technology for the identification of prostate cancer. Urol Oncol 2009 Jun 11. [Epub ahead of print].

10. Villers A, Lemaitre L, Haffner J, et al. Current status of MRI for the diagnosis, staging and prognosis of prostate cancer: implications for focal therapy and active surveillance. Curr Opin Urol 2009;19:274-82.

11. Onik G, Miessau M, Bostwick DG. Three-dimensional prostate mapping biopsy has a potentially significant impact on prostate cancer management. J Clin Oncol 2009;27:4321-6.

12. Wolff JM, Boeckmann $W$, Mattelaer $P$, et al. Determination of prostate gland volume by transrectal ultrasound: correlation with radical prostatectomy specimens. Eur Urol 1995;28:10-2.

13. Jani AB, Johnstone PA, Liauw SL, et al. Age and grade trends in prostate cancer (1974-2003): a Surveillance, Epidemiology, and End Results Registry analysis. Am I Clin Oncol 2008;31:375-8.

14. Bastian PJ, Mangold LA, Epstein Il, et al. Characteristics of insignificant clinical Tlc prostate tumors. A contemporary analysis. Cancer 2004;101:2001-5.

15. Latini DM, Hart SL, Knight SJ, et al. The relationship between anxiety and time to treatment for patients with prostate cancer on surveillance. J Urol 2007;178:826-31.

16. Polascik TJ, Mayes JM, Mouraviev V. Nerve-sparing focal cryoablation of prostate cancer. Curr Opin Urol 2009;19:182-7.

17. Eggener SE, Scardino PT, Carroll PR, et al. Focal therapy for localized prostate cancer: a critical appraisal of rationale and modalities. J Urol 2007;178:2260-7.

18. Lindner $U$, Weersink RA, Haider MA, et al. Image guided photothermal focal therapy for localized prostate cancer: phase I trial. J Urol 2009;182:1371-7.

19. Rubinsky J, Onik G, Mikus $P$, et al. Optimal parameters for the destruction of prostate cancer using irreversible electroporation. J Urol 2008;180:2668-74.

20. Rebillard X, Soulié M, Chartier-Kastler E, et al. High-intensity focused ultrasound in prostate cancer; a systematic literature review of the French Association of Urology. BJU Int 2008;101:1205-13.

21. Lambert EH, Bolte K, Masson P, et al. Focal cryosurgery: encouraging health outcomes for unifocal prostate cancer. Urology 2007;69:1117-20.

22. Tareen B, Godoy G, Sankin A, et al. Can contemporary transrectal prostate biopsy accurately select candidates for hemi-ablative focal therapy of prostate cancer? BJU Int 2009;104:195-9.

23. Scales CD Jr, Presti JC Jr, Kane CJ, et al. Predicting unilateral prostate cancer based on biopsy features: implications for focal ablative therapy-results from the SEARCH database. J Urol 2007;178:1249-52.

24. Bahn DK, Silverman P, Lee F Sr, et al. Focal prostate cryoablation: initial results show cancer control and potency preservation. J Endourol 2006;20:688-92.

25. Grover $S$, Srivastava A, Tan $G$, et al. Clinicopathological strategies to identify contralateral prostate cancer involvement in potential candidates for focal therapy. Int I Surg Pathol 2010;18:499-507.

26. Hofner T, Pfitzenmaier J, Arrabadi A,et al. PSA density lower cutoff value as a tool to exclude pathologic upstaging in initially diagnosed unilateral prostate cancer: impact on hemiablative focal therapy. World I Urol 2010 Dec 31. [Epub ahead of print].

Correspondence: Dr. Stavros Sfoungaristos, Patras University Hospital, Urology Department, Building A, 4th floor, Rion, Patras, 26500 Greece; fax: +302610993981; sfoungaristosst@gmail.com 